\section{The continued need to synthesize the results of genetic associations across multiple studies}

\section{To the Editor:}

The sequencing of the human genome and the increasing availability of high-throughput genotyping have led to an upsurge in published genetic associations. ${ }^{1}$ Identifying true genetic associations among the large volume of false positives has always been a difficult task. ${ }^{2}$ The use of systematic reviews, particularly meta-analyses, has been recommended to summarize and assess the cumulative evidence on genetic associations. ${ }^{3}$ Even in the era of genome-wide association studies (GWAS), meta-analysis has a role in synthesizing and integrating results of genetic association studies, ${ }^{4}$ yet it is not clear if these efforts have kept pace.

Using two applications included in the recently deployed Human Genome Epidemiology (HuGE) Navigator, ${ }^{5}$ HuGE Watch and HuGEpedia, we found that meta-analyses increased threefold as a proportion of all published articles on genetic associations from 2001 to 2007 (Table 1); however, meta-analyses still represent only a tiny fraction (3\%) of the genetic association literature. Among the 10 most frequently studied diseases (Table 2), genetic associations (e.g., APOE and Alzheimer disease) described in 25 or more primary research articles were far more likely to show at least one meta-analysis in the HuGEpedia (range, 47-100\%) than those described in 10 to 24 articles (range, $9-69 \%$ ) or in 5 to 9 articles (range, $0-32 \%)$.

The increasing popularity of GWAS reinforces, rather than diminishes, the importance of knowledge synthesis, including meta-analysis. ${ }^{4}$ In GWAS, identifying true-positive associations with genetic variants among hundreds of thousands tested is further complicated by the multistage designs used by many GWAS to test for replication of statistically significant results. ${ }^{6}$ Meta-analyses may be useful for integrating results across stages to identify true associations and could be used as prior knowledge in Bayesian approaches of selecting variants to carry forward to subsequent stages of analysis. ${ }^{7}$ Furthermore, as with candidate gene studies, meta-analyses of GWAS can be used to calculate more precise measures of effect and assess the heterogeneity and generalizability of genetic associations. ${ }^{4,8}$

HuGENet, a global collaboration of researchers dedicated to advancing public health genomics, has collaborated with 11 journals to promote the conduct of systematic reviews, including meta-analysis (HuGE Reviews). 3 Through December 2007, $65 \mathrm{HuGE}$ reviews have been published. Topics are registered with HuGENet and displayed on the HuGE Web site to help prevent duplicate efforts and encourage collaboration between the researchers. Guidance for conducting HuGE Reviews is provided in the HuGENet HuGE Review Handbook (http:// www.genesens.net/_intranet/doc_nouvelles/HuGE\%20Review\% 20 Handbook\%20v11.pdf). Authors are also encouraged to use recently published interim guidelines to assess the cumulative evidence for the genetic association of interest. ${ }^{9}$ Authors interested in selecting a topic for a HuGE Review can use the HuGE Navigator, as we did, to identify gaps in knowledge synthesis.

Describing and confirming genetic associations is a crucial first step in realizing the potential of genomic medicine. We show that although research on genetic associations is booming, more attention needs to be devoted to efforts to synthesize and interpret research findings. Meta-analyses are most likely to focus on frequently studied genetic associations; however, we did not find meta-analyses in the HuGE Navigator for many associations that have been studied 10 or more times. Assessing the cumulative evidence for associations is important in a field so plagued by nonreplication. ${ }^{2}$ We encourage investigators to perform and publish systematic reviews and meta-analyses of genetic associations and for journals to publish this work as part of the ongoing translation of gene discoveries into clinical medicine and public health.

Table 1

Articles reporting genetic associations, including meta-analyses, by year-HuGE Navigator knowledge base

\begin{tabular}{lrrrrrr}
\hline & 2001 & 2002 & 2003 & 2004 & 2005 & 2006 \\
\hline No. articles examining genetic associations & 2148 & 2776 & 2961 & 3645 & 4334 & 4808 \\
No. meta-analyses & 24 & 32 & 61 & 67 & 98 & 142 \\
Percent of meta-analyses & 1 & 1 & 2 & 2 & 2 & 3 \\
\hline
\end{tabular}

${ }^{a}$ As of December 12th, 2007. 


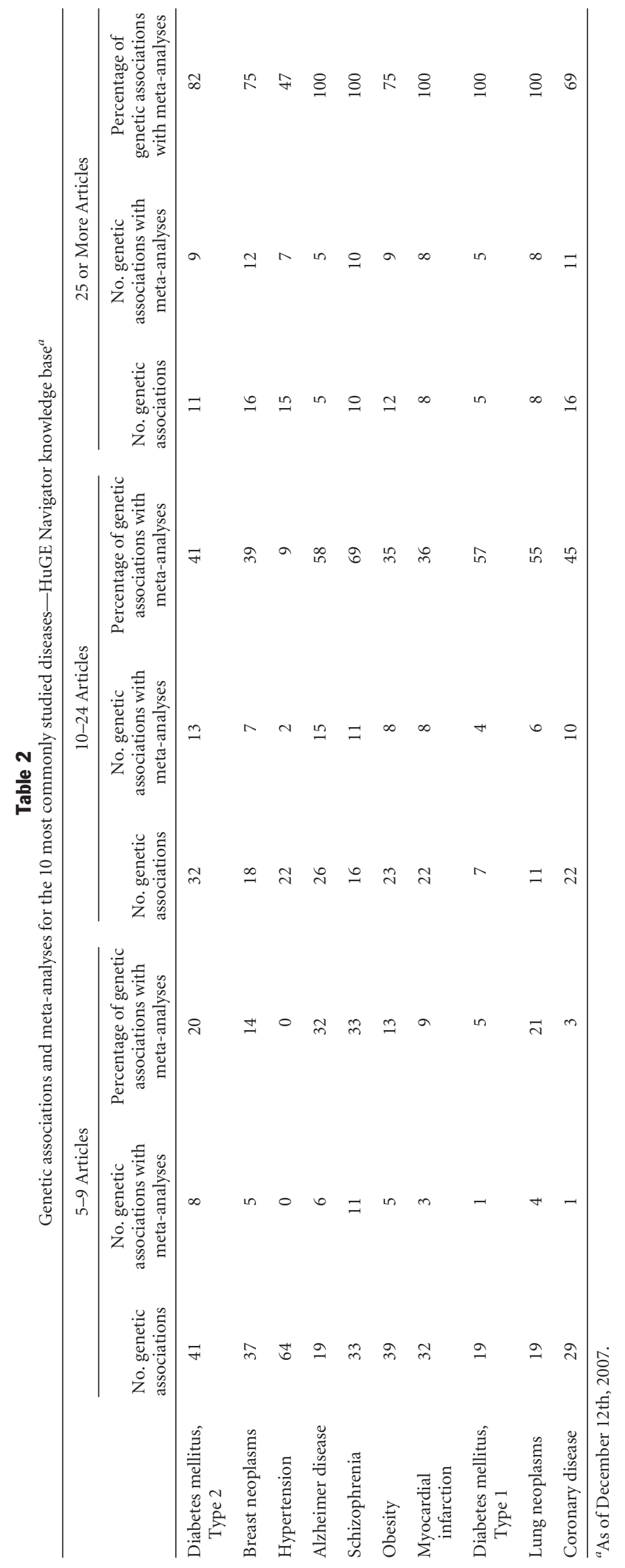


Ajay Yesupriya, MPH

Wei $\mathrm{Yu}, \mathrm{PhD}$

Melinda Clyne, MHS

Marta Gwinn, MD, MS

Muin J. Khoury, MD, PhD

National Office of Public Health Genomics Coordinating Center for Health Promotion

Centers for Disease Control and Prevention Atlanta, Georgia

Disclaimer: The findings and conclusions in this report are those of the authors and do not necessarily represent the views of the Centers for Disease Control and Prevention.

\section{References}

1. Lin BK, Clyne M, Walsh M, et al. Tracking the epidemiology of human genes in the literature: the HuGE Published Literature database. Am J Epidemiol 2006;164:1-4.
2. Ioannidis JP. Genetic associations: false or true? Trends Mol Med 2003;9: 135-138.

3. Ioannidis JP, Gwinn M, Little J, et al. A road map for efficient and reliable human genome epidemiology. Nat Genet 2006;38:3-5.

4. Evangelou E, Maraganore DM, Ioannidis JP. Meta-analysis in genome-wide association datasets: strategies and application in Parkinson disease. PLoS ONE 2007;2:e196.

5. Yu W, Gwinn M, Clyne M, Yesupriya A, Khoury MJ. A navigator for human genome epidemiology. Nat Genet 2008;40:124-125.

6. Wang H, Thomas DC, Pe'er I, Stram DO. Optimal two-stage genotyping designs for genome-wide association scans. Genet Epidemiol 2006;30:356-368.

7. Lewinger JP, Conti DV, Baurley JW, Triche TJ, Thomas DC. Hierarchical Bayes prioritization of marker associations from a genome-wide association scan for further investigation. Genet Epidemiol 2007;31:871-882.

8. Ioannidis JP, Patsopoulos NA, Evangelou E. Heterogeneity in meta-analyses of genome-wide association investigations. PLoS ONE 2007;2:e841.

9. Ioannidis JP, Boffetta P, Little J, et al. Assessment of cumulative evidence on genetic associations: interim guidelines. Int J Epidemiol 2008;37:120-132.

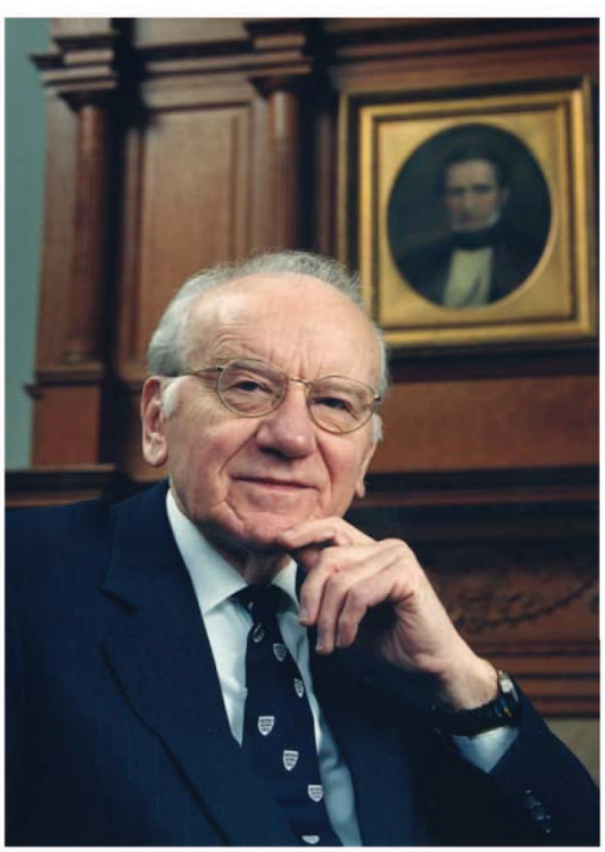

Photo by Keith Weller/Johns Hopkins Medicine

In memory of Victor A. McKusick, 1921-2008, whom we recently featured in our June issue of Genetics in Medicine.
A towering figure in genetics and one of the founders of our field. He will be missed. 